TAIWANESE JOURNAL OF MATHEMATICS

Vol. 16, No. 5, pp. 1613-1628, October 2012

This paper is available online at http://journal.taiwanmathsoc.org.tw

\title{
ON PERTURBATION OF LOCAL INTEGRATED COSINE FUNCTIONS
}

\author{
Chung-Cheng Kuo
}

\begin{abstract}
In this paper, we apply the contraction mapping theorem to obtain some bounded and unbounded perturbation theorems concerning local integrated cosine functions. Some growth conditions of perturbations of local integrated cosine functions are also established.
\end{abstract}

\section{INTRODUCTION}

Let $X$ be a Banach space with norm $\|\cdot\|$, and $L(X)$ denote the set of all bounded linear operators on $X$. For each $\alpha>0$ and $0<T_{0} \leq \infty$, a family $C(\cdot)(=\{C(t) \mid 0 \leq$ $\left.\left.t<T_{0}\right\}\right)$ in $L(X)$ is called a local $\alpha$-times integrated cosine function on $X$ if it is strongly continuous and satisfies

$$
\begin{aligned}
2 C(t) C(s) x= & \frac{1}{\Gamma(\alpha)}\left\{\left[\int_{0}^{t+s}-\int_{0}^{t}-\int_{0}^{s}\right](t+s-r)^{\alpha-1} C(r) x d r\right. \\
& +\int_{|t-s|}^{t}(s-t+r)^{\alpha-1} C(r) x d r \\
& +\int_{|t-s|}^{s}(t-s+r)^{\alpha-1} C(r) x d r \\
& \left.+\int_{0}^{|t-s|}(|t-s|+r)^{\alpha-1} C(r) x d r\right\}
\end{aligned}
$$

for all $x \in X$ and $0 \leq t, s \leq t+s<T_{0}$ (see [8-10, 12, 21]). Here $\Gamma(\cdot)$ denotes the Gamma function. Moreover, we say that $C(\cdot)$ is

(1.2) locally Lipschitz continuous if for each $0<t_{0}<T_{0}$ there exists a $K_{t_{0}}>0$ such that $\|C(t+h)-C(t)\| \leq K_{t_{0}} h$ for all $0 \leq t, h \leq t+h \leq t_{0}$;

(1.3) exponentially bounded if there exist $K, \omega \geq 0$ such that $\|C(t)\| \leq K e^{\omega t}$ for all $t \geq 0$;

Received September 30, 2010, accepted October 7, 2011.

Communicated by Chin-Cheng Lin.

2010 Mathematics Subject Classification: 47D60, 47D62.

Key words and phrases: Integrated cosine function, Generator, Abstract Cauchy problem.

Research partially supported by the National Science Council of Taiwan. Council of Taiwan. 
(1.4) exponentially Lipschitz continuous if there exist $K, \omega \geq 0$ such that $\| C(t+$ $h)-C(t) \| \leq K h e^{\omega(t+h)}$ for all $t, h \geq 0$;

(1.5) nondegenerate if $x=0$ whenever $C(t) x=0$ for all $0 \leq t<T_{0}$. In this case, the (integral) generator of $C(\cdot)$ is a linear operator $A$ in $X$ defined by $D(A)=$ $\left\{x \mid x, y_{x} \in X\right.$ and $C(t) x-j_{\alpha}(t) x=\int_{0}^{t} \int_{0}^{s} C(r) y_{x} d r d s$ for all $\left.0 \leq t<T_{0}\right\}$ and $A x=y_{x}$ for each $x \in D(A)$. Here $j_{\beta}(t)=\frac{t^{\beta}}{\Gamma(\beta+1)}$ for $\beta>-1$ and $t>0$.

In general,a local $\alpha$-times integrated cosine function is also called an $\alpha$-times integrated cosine function if $T_{0}=\infty$ (see $\left.[8,19,21]\right)$, an $\alpha$-times integrated cosine function may not be exponentially bounded (see [8]) and the generator of a nondegenerate local $\alpha$-times integrated cosine function may not be densely defined (see [2]). The formation of a local $\alpha$-times integrated cosine function is first constructed by the author in [8] for which $\alpha>0$ is arbitrary. Several examples concerning $\alpha$-times integrated cosine functions with densely defined generators are given as in [6], and in $[2,22]$ when integrated cosine functions are exponentially bounded. Perturbation of local $\alpha$-times integrated cosine functions is one of the subjects in the theory of cosine function, which has been extensively studied by many authors when considered integrated cosine functions are exponentially bounded (see [1,2])with $\alpha=0$ (see $[1,3,4,7,16]$ ) or $\alpha \in \mathbb{N}$ (see $[1,2,13,15-17,19,20])$. The purpose of this paper is to establish several bounded and unbounded perturbation theorems concerning local $\alpha$-times integarted cosine functions which generalize the classical perturbation result for cosine functions and may be done by using some results as in $[8,10]$ about the uniqueness of strong solutions of the following second order abstract Cauchy problem:

$$
A C P(A, f, x, y) \quad\left\{\begin{array}{l}
u^{\prime \prime}(t)=A u(t)+f(t) \quad \text { for } 0<t<T_{0} \\
u(0)=x, u^{\prime}(0)=y
\end{array}\right.
$$

where $x, y \in X$ are given, $A: D(A) \subset X \rightarrow X$ is a closed linear operator with domain $D(A)$ and range $R(A)$, and $f$ is an $X$-valued function defined on $\left(0, T_{0}\right)$. In section 2 , we first show that if $A$ generates a nondegenerate local $\alpha$-times integrated cosine function $C(\cdot)$ on $X$ and $B$ is a bounded linear operator from $\overline{D(A)}$ into $X$ such that $B x \in D\left(A^{l}\right)$ for all $x \in \overline{D(A)}$, then $A+B$ generates a nondegenerate local $\alpha$-times integrated cosine function $T(\cdot)$ on $X$ satisfying $T(\cdot) x=C(\cdot) x+D^{\alpha} S * B T(\cdot) x$ on $\left[0, T_{0}\right)$ for all $x \in X$ when

$$
\left\{\begin{aligned}
R(B) \subset D\left(A^{l}\right) & \text { if }[\alpha] \text { is even or }[\alpha] \text { is odd with } \alpha=[\alpha] \\
R(B) \subset D\left(A^{l+1}\right) & \text { if }[\alpha] \text { is odd with }[\alpha]<\alpha<[\alpha]+1
\end{aligned}\right.
$$

(see Theorem 2.11 below). Here $S(\cdot)=j_{0} * C(\cdot), l=\frac{[\alpha]}{2}$ if $[\alpha]$ is even, and $l=\frac{[\alpha]-1}{2}$ if $[\alpha]$ is odd. Moreover, $T(\cdot)$ is exponentially bounded (resp., norm continuous or exponentially Lipschitz continuous) if $C(\cdot)$ is. We then show that $T(\cdot)$ is also locally Lipschitz continuous if $C(\cdot)$ is and 


$$
\left\{\begin{array}{l}
R(B) \subset D\left(A^{l-1}\right) \text { if }[\alpha] \text { is even with } \alpha=[\alpha] \\
R(B) \subset D\left(A^{l}\right) \text { if }[\alpha] \text { is odd or }[\alpha] \text { is even with }[\alpha]<\alpha<[\alpha]+1
\end{array}\right.
$$

(see Theorem 2.13 below). In section 3, we first show that if $B$ is a bounded linear operator from $[D(A)]$ into $X$ such that $A+B$ is a closed linear operator from $D(A)$ into $X$, then $A+B$ generates a nondegenerate local $\alpha$-times integrated cosine function $T(\cdot)$ on $X$ satisfying $T(\cdot) x=C(\cdot) x+D^{\alpha+2} S * B \widetilde{\widetilde{T}}(\cdot) x$ on $\left[0, T_{0}\right)$ for all $x \in X$ when

$$
\left\{\begin{array}{l}
R(B) \subset D\left(A^{l+1}\right) \text { for all } x \in D(A) \text { if }[\alpha] \text { is even or }[\alpha] \text { is odd with } \alpha=[\alpha] \\
R(B) \subset D\left(A^{l+2}\right) \text { for all } x \in D(A) \text { if }[\alpha] \text { is odd with }[\alpha]<\alpha<[\alpha]+1
\end{array}\right.
$$

(see Theorem 3.1 below). Here $\widetilde{\widetilde{T}}(\cdot)=j_{1} * T(\cdot)$. Moreover, $T(\cdot)$ is exponentially bounded (resp., norm continuous or exponentially Lipschitz continuous) if $C(\cdot)$ is. We then show that $T(\cdot)$ is also locally Lipschitz continuous if $C(\cdot)$ is and

$$
\left\{\begin{array}{c}
R(B) \subset D\left(A^{l}\right) \text { if }[\alpha] \text { is even with } \alpha=[\alpha] \\
R(B) \subset D\left(A^{l+1}\right) \text { if }[\alpha] \text { is odd or }[\alpha] \text { is even with }[\alpha]<\alpha<[\alpha]+1
\end{array}\right.
$$

(see Theorem 3.2 below). We also show that if $B$ is a bounded linear operator from $[D(A)]$ into $X$ such that $A B=B A$ on $D\left(A^{2}\right)$, then $A+B$ generates a nondegenerate local $\alpha$-times integrated cosine function $T(\cdot)$ on $X$ satisfying $T(\cdot) x=C(\cdot) x+D^{\alpha} S *$ $(\lambda-A) B(\lambda-A)^{-1} T(\cdot) x$ on $\left[0, T_{0}\right)$ for all $x \in X$ when

$$
\left\{\begin{array}{l}
R(B) \subset D\left(A^{l+1}\right) \text { if }[\alpha] \text { is even or }[\alpha] \text { is odd with } \alpha=[\alpha] \\
R(B) \subset D\left(A^{l+2}\right) \text { if }[\alpha] \text { is odd with }[\alpha]<\alpha<[\alpha]+1
\end{array}\right.
$$

(see Corollary 3.5 below). Here $\lambda \in \rho(A)$ is fixed. Moreover, $T(\cdot)$ is exponentially bounded (resp., norm continuous or exponentially Lipschitz continuous) if $C(\cdot)$ is. We then show that $T(\cdot)$ is also locally Lipschitz continuous if $C(\cdot)$ is and

$$
\left\{\begin{array}{c}
R(B) \subset D\left(A^{l}\right) \text { if }[\alpha] \text { is even with } \alpha=[\alpha] \\
R(B) \subset D\left(A^{l+1}\right) \text { if }[\alpha] \text { is odd or }[\alpha] \text { is even with }[\alpha]<\alpha<[\alpha]+1
\end{array}\right.
$$

(see Corollary 3.6 below). An illustrative example concerning these theorems is also presented in the final part of this paper.

\section{Bounded Perturbation Theorems}

In this section, we first note some basic properties of a nondegenerate local $\alpha$-times integrated cosine function and known results about connections between the generator of a local $\alpha$-times integrated cosine functions for $\alpha>0$.

Proposition 2.1. (see $[8,10,14])$. Let $A$ be the generator of a nondegenerate local $\alpha$-times integrated cosine function $C(\cdot)$ on $X$. Then 
(2.1) $C(0)=0$ (the zero operator on $X)$;

(2.2) $A$ is closed and $\rho(A)$ (the resolvent set of $A$ ) is nonempty;

(2.3) $C(t) x \in D(A)$ and $C(t) A x=A C(t) x$ for $x \in D(A)$ and $0 \leq t<T_{0}$;

(2.4) $\int_{0}^{t} \int_{0}^{s} C(r) x d r d s \in D(A)$ and $A \int_{0}^{t} \int_{0}^{s} C(r) x d r d s=C(t) x-j_{\alpha}(t) x$ for $x \in X$ and $0 \leq t<T_{0}$;

(2.5) $R(C(t)) \subset \overline{D(A)}$ for $0 \leq t<T_{0}$;

(2.6) for each $\beta>\alpha, j_{\beta-\alpha-1} * C(\cdot)$ is a nondegenerate local $\beta$-times integrated cosine function on $X$ with generator $A$.

In the following, we shall always assume that $\alpha>0, l=\frac{[\alpha]}{2}$ if $[\alpha]$ is even, and $l=\frac{[\alpha]-1}{2}$ if $[\alpha]$ is odd.

Definition 2.2. Let $k=[\alpha]+1$, and $I$ be a subinterval of $\left[0, T_{0}\right)$ containing $\{0\}$. For each $v: I \rightarrow X$, we write $v \in C^{\alpha}(I, X)$ if $v=v(0)+j_{\alpha-k} * u$ on $I$ for some $u \in C^{k-1}(I, X)$. In this case,we say that $v$ is $\alpha$-times continuously differetiable on I and the $(k-1)$ th order derivative $u^{(k-1)}$ of $u$ on I is called the $\alpha$ th order derivative of $v$ on I and denoted by $D^{\alpha} v$ (on I) or $D^{\alpha} v: I \rightarrow X$. Here $C^{k}(I, X)$ denotes the set of all $k$-times continuously differetiable functions from $I$ into $X, C^{0}(I, X)=C(I, X)$ the set of all continuous functions from $I$ into $X$, and $f * g(t)=\int_{0}^{t} f(t-s) g(s) d s$ for each function $f$ from $I$ into $\mathbb{C}$ (or into $L(X)$ ) and each function $g$ from $I$

Remark 2.3. Let $k=[\alpha]+1$, and $v \in C^{\alpha}(I, X)$ for some subinterval $I$ of $\left[0, T_{0}\right)$ containing $\{0\}$. Assume that $v(0)=0$. Then $j_{k-\alpha-1} * v \in C^{k}(I, X), v \in C^{\alpha-i}(I, X)$ and $D^{\alpha-i} v=\left(j_{k-\alpha-1} * v\right)^{(k-i)}$ on $I$ for all integers $0 \leq i \leq k-1$. In particular, $j_{\alpha}(\cdot) \in C^{\alpha}\left(\left[0, T_{0}\right), \mathbb{C}\right)$ and $D^{\alpha-i} j_{\alpha}(\cdot)=D^{k-i} j_{k}(\cdot)=j_{i}(\cdot)$ on $\left[0, T_{0}\right)$ for all integers $0 \leq i \leq k-1$.

Definition 2.4. Let $A: D(A) \subset X \rightarrow X$ be a closed linear operator in $X$ with domain $D(A)$ and range $R(A)$. A function $u:\left[0, T_{0}\right) \rightarrow X$ is called a (strong) solution of $A C P(A, f, x, y)$ if $u \in C^{2}\left(\left(0, T_{0}\right), X\right) \cap C^{1}\left(\left[0, T_{0}\right), X\right) \cap C\left(\left(0, T_{0}\right),[D(A)]\right)$ and satisfies $A C P(A, f, x, y)$. Here $[D(A)]$ denotes the Banach space $D(A)$ with norm $|\cdot|$ defined by $|x|=\|x\|+\|A x\|$ for $x \in D(A)$.

Remark 2.5. $u \in C\left(\left[0, T_{0}\right),[D(A)]\right)$, if $f \in C\left(\left[0, T_{0}\right), X\right)$ and $u$ is a (strong) solution of $A C P(A, f, x, y)$ in $C^{2}\left(\left[0, T_{0}\right), X\right)$.

Theorem 2.6 (see $[8,10])$. A generates a nondegenerate local $\alpha$-times integrated $C$-cosine function $C(\cdot)$ on $X$ if and only if for each $x \in X, A C P\left(A, j_{\alpha}(\cdot) x, 0,0\right)$ has a unique (strong) solution $u(\cdot, x)$ in $C^{2}\left(\left[0, T_{0}\right), X\right)$. In this case, we have $u(\cdot, x)=$ $j_{1} * C(\cdot) x$ for all $x \in X$. 
Proposition 2.7. (see [10]). Let $A$ be the generator of a nondegenerate local $\alpha$-times integrated cosine function $C(\cdot)$ on $X, x, y \in X$ and $f \in L_{l o c}^{1}\left(\left[0, T_{0}\right), X\right) \cap$ $C\left(\left(0, T_{0}\right), X\right)$. Then $A C P(A, C f, C x, C y)$ has a (strong) solution u in $C^{2}\left(\left[0, T_{0}\right), X\right)$ if and only if $v(\cdot)=C(\cdot) x+S(\cdot) y+S * f(\cdot) \in C^{\alpha+2}\left(\left[0, T_{0}\right), X\right)$. In this case, $u=D^{\alpha} v$ on $\left[0, T_{0}\right)$. Here $S(\cdot)=j_{0} * C(\cdot)$.

Lemma 2.8. (see [11]). Let $V(\cdot)$ and $Z(\cdot)$ be strongly continuous families of bounded linear operators from $X$ into $Y$ for some Banach space $Y$, and let $W(\cdot)$ be a strongly continuous family in $L(Y)$ such that $Z(\cdot) x=V(\cdot) x+W * Z(\cdot) x$ on $\left[0, T_{0}\right)$ for all $x \in X$. Then $Z(\cdot)$ is exponentially bounded (resp., norm continuous or exponentially Lipschitz continuous) if $V(\cdot)$ and $W(\cdot)$ both are.

Lemma 2.9. (see [11]). Let $V(\cdot)$ be a locally Lipschitz continuous family of bounded linear operators from $X$ into $Y$ for some Banach space $Y$, and let $W(\cdot)$ be a locally Lipschitz continuoous family in $L(Y)$ with $W(0)=0$ on $Y$. Then there exists a unique locally Lipschitz continuous family $Z(\cdot)$ of bounded linear operators from $X$ into $Y$ such that

$$
Z(t) x=V(t) x+\frac{d}{d t} W * Z(t) x
$$

for all $x \in X$ and $0 \leq t<T_{0}$.

Combining (2.3) with (2.4) we can obtain the next result by induction.

Remark 2.10. Let $A$ be the generator of a nondegenerate local $\alpha$-times integrated cosine function $C(\cdot)$ on $X$. Then for each $m \in \mathbb{N}$ and $x \in D\left(A^{m}\right)$,

$$
C(\cdot) x=j_{2(m-1)} * S(\cdot) A^{m} x+\sum_{i=0}^{m-1} j_{\alpha+2 i}(\cdot) A^{i} x \text { on }\left[0, T_{0}\right) .
$$

The following two theorems are the main results of this section concerning bounded perturbation of local $\alpha$-times integrated cosine functions on $X$. The first one has been obtained by Zheng in [20] when $\alpha \in \mathbb{N}$ and $B$ is a bounded linear operator on $X$ except for the growth condition of $T(\cdot)$.

Theorem 2.11. Let $A$ be the generator of a nondegenerate local $\alpha$-times integrated cosine function $C(\cdot)$ on $X$. Assume that $B$ is a bounded linear operator from $\overline{D(A)}$ into $X$. Then $A+B$ generates a nondegenerate local $\alpha$-times integrated cosine function $T(\cdot)$ on $X$ satisfying

$$
T(\cdot) x=C(\cdot) x+D^{\alpha} S * B T(\cdot) x \text { on }\left[0, T_{0}\right)
$$

for all $x \in X$ when

$$
\left\{\begin{aligned}
R(B) \subset D\left(A^{l}\right) \text { if }[\alpha] \text { is even or }[\alpha] \text { is odd with } \alpha=[\alpha] \\
R(B) \subset D\left(A^{l+1}\right) \text { if }[\alpha] \text { is odd with }[\alpha]<\alpha<[\alpha]+1 .
\end{aligned}\right.
$$


Moreover, $T(\cdot)$ is exponentially bounded (resp., norm continuous or exponentially Lipschitz continuous) if $C(\cdot)$ is.

Proof. Clearly, $A+B: D(A) \subset X \rightarrow X$ is closed. Now if $\alpha \in \mathbb{N}, 0<t_{0}<T_{0}$ and $f \in C\left(\left[0, t_{0}\right], \overline{D(A)}\right)$ are fixed. By (2.7), (2.9) and Remark 2.3, we have $S * B f \in$ $C^{\alpha}\left(\left[0, t_{0}\right], \overline{D(A)}\right)$ and

$$
\begin{aligned}
& D^{\alpha} S * B f(t) \\
& \qquad \begin{array}{ll}
C * B f(t) & \text { if } \alpha=1 \\
S * A^{l} B f(t)+\sum_{i=0}^{l-1} j_{2 i+1} * A^{i} B f(t) & \text { if } \alpha \text { is even and } \alpha \geq 2 \\
C * A^{l} B f(t)+\sum_{i=0}^{l-1} j_{2 i+1} * A^{i} B f(t) & \text { if } \alpha \text { is odd and } \alpha \geq 3
\end{array}
\end{aligned}
$$

for all $0 \leq t \leq t_{0}$. Next if $[\alpha]<\alpha<[\alpha]+1$. We set $\widetilde{C}(\cdot)=j_{[\alpha]-\alpha} * C(\cdot)$ and $\widetilde{S}(\cdot)=j_{0} * \widetilde{C}(\cdot)$, then $\widetilde{C}(\cdot)$ is $([\alpha]+1)$-times integrated $C$-cosine function on $X$ with generator $A$. Just as in the case $\alpha \in \mathbb{N}$, we also have $D^{\alpha} S * B f \in C^{\alpha}\left(\left[0, t_{0}\right], \overline{D(A)}\right)$ and

$$
\begin{aligned}
& D^{\alpha} S * B f(t) \\
= & D^{[\alpha]+1} \widetilde{S} * B f(t) \\
= & D^{[\alpha]} \widetilde{C} * B f(t) \\
= & \left\{\begin{array}{l}
\widetilde{C} * A^{l} B f(t)+\sum_{i=0}^{l-1} j_{2 i+1} * A^{i} B f(t) \quad \text { if }[\alpha] \text { is even and }[\alpha] \geq 2 \\
\widetilde{S} * A^{l+1} B f(t)+\sum_{i=0}^{l} j_{2 i+1} * A^{i} B f(t) \text { if }[\alpha] \text { is odd }
\end{array}\right.
\end{aligned}
$$

on $\left[0, t_{0}\right]$ for all $0<t_{0}<T_{0}$ and $f \in C\left(\left[0, t_{0}\right], \overline{D(A)}\right)$. We shall show that for each $x \in X$ there exists a function $w_{x}$ in $C\left(\left[0, T_{0}\right), \overline{D(A)}\right)$ such that $w_{x}(\cdot)=C(\cdot) x+$ $D^{\alpha} S * B w_{x}(\cdot)$ on $\left[0, T_{0}\right)$. Indeed, fix $x \in X$ and $0<t_{0}<T_{0}$ we define $U$ : $C\left(\left[0, t_{0}\right], \overline{D(A)}\right) \rightarrow C\left(\left[0, t_{0}\right], \overline{D(A)}\right)$ by $U(f)(\cdot)=C(\cdot) x+D^{\alpha}(S * B f)(\cdot)$ on $\left[0, t_{0}\right]$ for all $f \in C\left(\left[0, t_{0}\right], \overline{D(A)}\right)$. From (2.1), (2.5) and the assumption $B x \in D\left(A^{\lambda}\right)$ for all $x \in \overline{D(A)}$, we have that $U$ is well-defined and $A^{i} B$ is a bounded linear operator from $\overline{D(A)}$ into $X$ for all integers $0 \leq i \leq \lambda$. Here $\lambda=l$ if $[\alpha]$ is odd or $[\alpha]$ is even with $[\alpha]<\alpha<[\alpha]+1$, and $\lambda=l-1$ if $[\alpha]$ is even with $\alpha=[\alpha]$. We first claim that

$$
\left\|D^{\alpha} S * B f(t)\right\| \leq M_{t_{0}} \int_{0}^{t}\|f(s)\| d s
$$

for all $f \in C\left(\left[0, t_{0}\right], \overline{D(A)}\right)$ and $0 \leq t \leq t_{0}$, where 


$$
M_{t_{0}}= \begin{cases}\sup _{0 \leq r \leq t_{0}}\|S(r)\|\left\|A^{l} B\right\|+\sum_{i=0}^{l-1} j_{2 i+1}\left(t_{0}\right)\left\|A^{i} B\right\| & \text { if } 2 \leq \alpha \in \mathbb{N} \text { is even } \\ \sup _{0 \leq r \leq t_{0}}\|C(r)\|\|B\| & \text { if } \alpha=1 \\ \sup _{0 \leq r \leq t_{0}}\|C(r)\|\left\|A^{l} B\right\|+\sum_{i=0}^{l-1} j_{2 i+1}\left(t_{0}\right)\left\|A^{i} B\right\| & \text { if } 2 \leq \alpha \in \mathbb{N} \text { is odd } \\ \sup _{0 \leq r \leq t_{0}}\|\widetilde{C}(r)\|\|B\| & \text { if } 0<\alpha<1 \\ \sup _{0 \leq r \leq t_{0}}\|\widetilde{C}(r)\|\left\|A^{l} B\right\|+\sum_{i=0}^{l-1} j_{2 i+1}\left(t_{0}\right)\left\|A^{i} B\right\| & \text { if }[\alpha]<\alpha<[\alpha]+1 \\ \sup _{0 \leq r \leq t_{0}}\|\widetilde{S}(r)\|\left\|A^{l+1} B\right\|+\sum_{i=0}^{l} j_{2 i+1}\left(t_{0}\right)\left\|A^{i} B\right\| & \text { if }[\alpha]<\alpha<[\alpha]+1\end{cases}
$$

To this end, we consider only the case $\alpha \in \mathbb{N}$, for the case $[\alpha]<\alpha<[\alpha]+1$ can be treated similarly. Indeed, if $\alpha \in \mathbb{N}$ is even and $f \in C\left(\left[0, t_{0}\right], \overline{D(A)}\right)$, then

$$
\begin{aligned}
\left\|S * A^{l} B f(t)\right\| & \leq \int_{0}^{t}\left\|S(t-s) A^{l} B f(s)\right\| d s \\
& \leq \int_{0}^{t} \sup _{0 \leq r \leq t_{0}}\|S(r)\|\left\|A^{l} B f(s)\right\| d s \\
& =\sup _{0 \leq r \leq t_{0}}\|S(r)\|\left\|A^{l} B\right\| \int_{0}^{t}\|f(s)\| d s
\end{aligned}
$$

and

$$
\begin{aligned}
\left\|j_{2 i+1} * A^{i} B f(t)\right\| & \leq \int_{0}^{t}\left\|j_{2 i+1}(t-s) A^{i} B f(s)\right\| d s \\
& \leq \int_{0}^{t} j_{2 i+1}\left(t_{0}\right)\left\|A^{i} B\right\|\|f(s)\| d s \\
& =j_{2 i+1}\left(t_{0}\right)\left\|A^{i} B\right\| \int_{0}^{t}\|f(s)\| d s
\end{aligned}
$$

for all $0 \leq t \leq t_{0}$ and integers $0 \leq i \leq l-1$. By (2.10), (2.13) and (2.14), we have

$$
\left\|D^{\alpha} S * B f(t)\right\| \leq\left\|S * A^{l} B f(t)\right\|+\sum_{i=0}^{l-1}\left\|j_{2 i+1} * A^{i} B f(t)\right\| \leq M_{t_{0}} \int_{0}^{t}\|f(s)\| d s
$$

for all $0 \leq t \leq t_{0}$, that is, (2.12) holds when $\alpha \in \mathbb{N}$ is even. Similarly, we can show that (2.12) also holds when $\alpha \in \mathbb{N}$ is odd. Having shown that (2.12) holds. By induction, we also have 


$$
\begin{aligned}
\left\|U^{n} f(t)-U^{n} g(t)\right\| & =\left\|U\left(U^{n-1} f\right)(t)-U\left(U^{n-1} g\right)(t)\right\| \\
& =\left\|D^{\alpha} S * B\left(U^{n-1} f-U^{n-1} g\right)(t)\right\| \\
& \leq M_{t_{0}}^{n} \int_{0}^{t} j_{n-1}(t-s)\|f(s)-g(s)\| d s \\
& \leq M_{t_{0}}^{n} j_{n}(t)\|f-g\| \\
& \leq M_{t_{0}}^{n} j_{n}\left(t_{0}\right)\|f-g\|
\end{aligned}
$$

for all $f, g \in C\left(\left[0, t_{0}\right], \overline{D(A)}\right), 0 \leq t \leq t_{0}$ and $n \in \mathbb{N}$, where $\|f-g\|=\max _{0 \leq s \leq t_{0}} \| f(s)-$ $g(s) \|$. Since $M_{t_{0}}^{n} j_{n}\left(t_{0}\right) \rightarrow 0$ as $n \rightarrow \infty$, we obtain from the contraction mapping theorem that there exists a unique function $w_{x, t_{0}}$ in $C\left(\left[0, t_{0}\right], \overline{D(A)}\right)$ such that $w_{x, t_{0}}(\cdot)=$ $C(\cdot) x+D^{\alpha} S * B w_{x, t_{0}}(\cdot)$ on $\left[0, t_{0}\right]$. In this case, we set $w_{x}(t)=w_{x, t_{0}}(t)$ for all $0 \leq t \leq t_{0}<T_{0}$, then $w_{x}(\cdot)$ is a unique function in $C\left(\left[0, T_{0}\right), \overline{D(A)}\right)$ such that $w_{x}(\cdot)=$ $C(\cdot) x+D^{\alpha} S * B w_{x}(\cdot)$ on $\left[0, T_{0}\right)$. Since $S * j_{\alpha}(\cdot) x+S * j_{1} * B w_{x}(\cdot) \in C^{\alpha+2}\left(\left[0, T_{0}\right), X\right)$ and $D^{\alpha}\left(S * j_{\alpha}(\cdot) x+S * j_{1} * B w_{x}(\cdot)\right)=j_{0} * S(\cdot) x+j_{1} * D^{\alpha} S * B w_{x}(\cdot)=j_{1} * w_{x}(\cdot)$ on $\left[0, T_{0}\right)$, we obtain from Proposition 2.7 that $u=j_{1} * w_{x}$ is the unique (strong) solution of $A C P\left(A, j_{\alpha}(\cdot) x+j_{1} * B w_{x}(\cdot), 0,0\right)$ in $C^{2}\left(\left[0, T_{0}\right), X\right)$, and so $u=j_{1} * w_{x}$ is the unique function in $C^{2}\left(\left[0, T_{0}\right), X\right)$ such that $u^{\prime \prime}(\cdot)\left(=A u+j_{\alpha}(\cdot) x+j_{1} * B w_{x}(\cdot)=\right.$ $\left.A u+j_{\alpha}(\cdot) x+j_{1} * B w_{x}(\cdot)=A u(\cdot)+j_{\alpha}(\cdot) x+B u(\cdot)\right)=(A+B) u(\cdot)+j_{\alpha}(\cdot) x$ on $\left[0, T_{0}\right)$. Hence $u=j_{1} * w_{x}$ is a unique solution of $A C P\left(A+B, j_{\alpha}(\cdot) x, 0,0\right)$ in $C^{2}\left(\left[0, T_{0}\right), X\right)$, which together with Theorem 2.6 implies that $A+B$ generates a nondegenerate local $\alpha$-times integrated cosine function $T(\cdot)$ on $X$ satisfying (2.8). We conclude from (2.10), (2.11) and Lemma 2.8 that $T(\cdot)$ is also exponentially bounded (resp., exponentially Lipschitz continuous) if $C(\cdot)$ is, by setting $Y=\overline{D(A)}=C(\cdot)$, $Z(\cdot)=T(\cdot)$ and

$$
W(\cdot)= \begin{cases}S(\cdot) A^{l} B+\sum_{i=0}^{l-1} j_{2 i+1}(\cdot) A^{i} B & \text { if } \alpha \in \mathbb{N} \text { is even } \\ C(\cdot) B & \text { if } \alpha=1 \\ C(\cdot) A^{l} B+\sum_{i=0}^{l-1} j_{2 i+1}(\cdot) A^{i} B & \text { if } \alpha \in \mathbb{N} \text { is odd } \\ \widetilde{C}(\cdot) B & \text { if } 0<\alpha<1 \\ \widetilde{C}(\cdot) A^{l} B+\sum_{i=0}^{l-1} j_{2 i+1}(\cdot) A^{i} B & \text { if }[\alpha]<\alpha<[\alpha]+1 \text { and }[\alpha] \text { is even } \\ \widetilde{S}(\cdot) A^{l+1} B+\sum_{i=0}^{l} j_{2 i+1}(\cdot) A^{i} B & \text { if }[\alpha]<\alpha<[\alpha]+1 \text { and }[\alpha] \text { is odd }\end{cases}
$$

in Lemma 2.8. 
Remark 2.12. (see [11]). Let $W(\cdot)$ be a locally Lipschitz continuous family in $L(Y)$ with $W(0)=0$ on $Y$ for some Banach space $Y$ and $g \in L_{l o c}^{1}\left(\left[0, T_{0}\right), Y\right)$. Then $W * g \in C^{1}\left(\left[0, T_{0}\right), Y\right)$ and for each $0<t_{0}<T_{0}$, we have $\left\|(W * g)^{\prime}(t)\right\| \leq$ $K_{t_{0}} \int_{0}^{t}\|g(s)\| d s$ for all $0 \leq t \leq t_{0}$. Here $K_{t_{0}}$ is given as in (1.2) with $C(\cdot)$ is replaced by $W(\cdot)$. Moreover, $(W * g)^{\prime}(\cdot)$ is locally Lipschitz continuous if $g$ is.

Theorem 2.13. Let $A$ be the generator of a locally Lipschitz continuous nondegenerate local $\alpha$-times integrated cosine function $C(\cdot)$ on $X$ for some $\alpha \geq 1$. Assume that $B$ is a bounded linear operator from $\overline{D(A)}$ into $X$. Then $A+B$ generates a locally Lipschitz continuous nondegenerate local $\alpha$-times integrated cosine function $T(\cdot)$ on $X$ satisfying (2.8) when either $\alpha=1$, or $\alpha>1$ and

$$
\left\{\begin{array}{l}
R(B) \subset D\left(A^{l-1}\right) \text { if }[\alpha] \text { is even and } \alpha=[\alpha] \in \mathbb{N} \\
R(B) \subset D\left(A^{l}\right) \text { if }[\alpha] \text { is odd or }[\alpha] \text { is even with }[\alpha]<\alpha<[\alpha]+1
\end{array}\right.
$$

Just as in the proof of Theorem 2.11, we shall first show that that $A+B$ generates a nondegenerate local $\alpha$-times integrated cosine function $T(\cdot)$ on $X$ satisfying (2.8) and need only to show that for each $0<t_{0}<T_{0}$, we have $\left\|D^{\alpha} S * B f(t)\right\| \leq$ $N_{t_{0}} \int_{0}^{t}\|f(s)\| d s$ for all $f \in C\left(\left[0, t_{0}\right], \overline{D(A)}\right)$ and $0 \leq t \leq t_{0}$ when $\alpha>1$. Here

$$
N_{t_{0}}= \begin{cases}K_{t_{0}}\|B\| & \text { if } \alpha=2 \\ K_{t_{0}}\left\|A^{l-1} B\right\|+\sum_{i=0}^{l-2} j_{2 i+1}\left(t_{0}\right)\left\|A^{i} B\right\| & \text { if } \alpha \in \mathbb{N} \text { is even and } \alpha \geq 4 \\ \sup _{0 \leq r \leq t_{0}}\|C(r)\|\left\|A^{l} B\right\|+\sum_{i=0}^{l-1} j_{2 i+1}\left(t_{0}\right)\left\|A^{i} B\right\| & \text { if } \alpha \in \mathbb{N} \text { is odd and } \alpha \geq 3 \\ \sup _{0 \leq r \leq t_{0}}\|\widetilde{C}(r)\|\left\|A^{l} B\right\|+\sum_{i=0}^{l-1} j_{2 i+1}\left(t_{0}\right)\left\|A^{i} B\right\| & \text { if }[\alpha]<\alpha<[\alpha]+1 \\ K_{t_{0} j_{2-\alpha}\left(t_{0}\right)\|B\|} & \text { and }[\alpha] \text { is even } \\ K_{t_{0}} j_{[\alpha]+1-\alpha}\left(t_{0}\right)\left\|A^{l} B\right\|+\sum_{i=0}^{l-1} j_{2 i+1}\left(t_{0}\right)\left\|A^{i} B\right\| & \text { if }[\alpha]<\alpha<[\alpha]+1 \\ & \text { and }[\alpha] \text { is odd }\end{cases}
$$

and $K_{t_{0}}$ is given as in (1.2). Indeed, if $0<t_{0}<T_{0}$ is given. The local Lipschitz continuity of $C(\cdot)$ implies that $\widetilde{C}(\cdot)$ is locally Lipschitz continuous with a Lipschitz constant $K_{t_{0}} j_{[\alpha]+1-\alpha}\left(t_{0}\right)$ on $\left[0, t_{0}\right]$. Combining this, Remarks 2.10 and 2.12 with (2.17), we have $S * B f \in C^{\alpha}\left(\left[0, t_{0}\right], \overline{D(A)}\right)$ and 


$$
\begin{aligned}
& D^{\alpha} S * B f(t)= \\
& \begin{cases}\frac{d}{d t} C * B f(t) & \text { if } \alpha=2 \\
\frac{d}{d t} C * A^{l-1} B f(t)+\sum_{i=0}^{l-2} j_{2 i+1} * A^{i} B f(t) & \text { if } \alpha \in \mathbb{N} \text { is even and } \alpha \geq 4 \\
C * A^{l} B f(t)+\sum_{i=0}^{l-1} j_{2 i+1} * A^{i} B f(t) & \text { if } \alpha \in \mathbb{N} \text { is odd and } \alpha \geq 3 \\
\widetilde{C} * A^{l} B f(t)+\sum_{i=0}^{l-1} j_{2 i+1} * A^{i} B f(t) & \text { if }[\alpha] \text { is even and }[\alpha]<\alpha<[\alpha]+1 \\
\frac{d}{d t} \widetilde{C} * B f(t) & \text { if } 1<\alpha<2 \\
\frac{d}{d t} \widetilde{C} * A^{l} B f(t)+\sum_{i=0}^{l-1} j_{2 i+1} * A^{i} B f(t) & \text { if }[\alpha] \text { is odd and }[\alpha]<\alpha<[\alpha]+1 .\end{cases}
\end{aligned}
$$

By Remark 2.12, we also have $\left\|\frac{d}{d t} C * A^{l-1} B f(t)\right\| \leq K_{t_{0}}\left\|A^{l-1} B\right\| \int_{0}^{t}\|f(s)\| d s$ for all $f \in C\left(\left[0, t_{0}\right], \overline{D(A)}\right)$ and $0 \leq t \leq t_{0}$ if $\alpha \in \mathbb{N}$ is even with $\alpha \geq 2$, and $\| \frac{d}{d t} \widetilde{C} *$ $A^{l} B f(t)\left\|\leq K_{t_{0}} j_{[\alpha]+1-\alpha}\right\| A^{l} B\left\|\int_{0}^{t}\right\| f(s) \| d s$ for all $f \in C\left(\left[0, t_{0}\right], \overline{D(A)}\right)$ and $0 \leq$ $t \leq t_{0}$ if $[\alpha]$ is odd with $[\alpha]<\alpha<[\alpha]+1$. Consequently, $\left\|D^{\alpha} S * B f(t)\right\| \leq$ $N_{t_{0}} \int_{0}^{t}\|f(s)\| d s$ when $\alpha>1$. Having shown that $A+B$ generates a nondegenerate local $\alpha$-times integrated cosine function $T(\cdot)$ on $X$ satisfying (2.8). We conclude from Lemma 2.9 and (2.18) that $T(\cdot)$ is also locally Lipschitz continuous: it suffices to set $Y=\overline{D(A)}, V(\cdot)=C(\cdot), Z(\cdot)=T(\cdot)$ and

$$
W(\cdot)= \begin{cases}C(\cdot) B & \text { if } \alpha=2 \\ C(\cdot) A^{l-1} B+\sum_{i=0}^{l-2} j_{2 i+2}(\cdot) A^{i} B & \text { if } \alpha \in \mathbb{N} \text { is even and } \alpha \geq 4 \\ S(\cdot) A^{l} B+\sum_{i=0}^{l-1} j_{2 i+2}(\cdot) A^{i} B & \text { if } \alpha \in \mathbb{N} \text { is odd and } \alpha \geq 3 \\ \widetilde{S}(\cdot) A^{l} B+\sum_{i=0}^{l-1} j_{2 i+2}(\cdot) A^{i} B & \text { if }[\alpha] \text { is even and }[\alpha]<\alpha<[\alpha]+1 \\ \widetilde{C}(\cdot) B & \text { if } 1<\alpha<2 \\ \widetilde{C}(\cdot) A^{l} B+\sum_{i=0}^{l-1} j_{2 i+2}(\cdot) A^{i} B & \text { if }[\alpha] \text { is odd and }[\alpha]<\alpha<[\alpha]+1\end{cases}
$$

in Lemma 2.9. 


\section{Unbounded Perturbation Theorems}

By slightly modifying the proof of Theorem 2.11, we can establish the following unbounded perturbation theorems concerning local $\alpha$-times integrated cosine functions on $X$.

Theorem 3.1. Let $C(\cdot)$ be a nondegenerate local $\alpha$-times integrated cosine function on $X$ with generator $A$. Assume that $B$ is a bounded linear operator from $[D(A)]$ into $X$ such that $A+B$ is a closed linear operator from $D(A)$ into $X$. Then $A+B$ generates a nondegenerate local $\alpha$-times integrated cosine function $T(\cdot)$ on $X$ satisfying

$$
T(\cdot) x=C(\cdot) x+D^{\alpha+2} S * B \widetilde{\widetilde{T}}(\cdot) x \quad \text { on }\left[0, T_{0}\right)
$$

for all $x \in X$ when

$$
\left\{\begin{array}{l}
R(B) \subset D\left(A^{l+1}\right) \text { if }[\alpha] \text { is even or }[\alpha] \text { is odd with } \alpha=[\alpha] \\
R(B) \subset D\left(A^{l+2}\right) \text { if }[\alpha] \text { is odd with }[\alpha]<\alpha<[\alpha]+1
\end{array}\right.
$$

Here $\widetilde{T}(\cdot)=j_{1} * T(\cdot)$. Moreover, $T(\cdot)$ is also exponentially bounded (resp., norm continuous or exponentially Lipschitz continuous) if $C(\cdot)$ is.

Proof. We consider only the case $\alpha \in \mathbb{N}$, for the case $[\alpha]<\alpha<[\alpha]+1$ can be treated similarly. Just as in the proof of Theorem 2.11, for each $0<t_{0}<T_{0}$, we can apply (2.10) and the fact $B x \in D\left(A^{l+1}\right)$ for all $x \in D(A)$ to establish the following inequalities as with (2.12)-(2.15):

$$
\begin{array}{r}
\left|S * A^{l} B f(t)\right| \leq \sup _{0 \leq r \leq t_{0}}\|S(r)\|\left|A^{l} B\right| \int_{0}^{t}|f(s)| d s, \\
\left|j_{2 i+1} * A^{i} B f(t)\right| \leq j_{2 i+1}\left(t_{0}\right)\left|A^{i} B\right| \int_{0}^{t}|f(s)| d s
\end{array}
$$

for all $0 \leq t \leq t_{0}$ and integers $0 \leq i \leq l-1$,

$$
\left|D^{\alpha} S * B f(t)\right| \leq M_{t_{0}} \int_{0}^{t}|f(s)| d s
$$

for all $0 \leq t \leq t_{0}$, and

$$
\left|U^{n} f(t)-U^{n} g(t)\right| \leq M_{t_{0}}^{n} j_{n}\left(t_{0}\right)|f-g|
$$

for all $f, g \in C\left(\left[0, t_{0}\right],[D(A)]\right), 0 \leq t \leq t_{0}$ and $n \in \mathbb{N}$ is even. Here $\left|A^{i} B\right|$ denotes the norm of $A^{i} B$ in $L([D(A)])$ for all integers $0 \leq i \leq l,|f-g|=\max _{0 \leq s \leq t_{0}}|f(s)-g(s)|$ 
and $U: C\left(\left[0, t_{0}\right],[D(A)]\right) \rightarrow C\left(\left[0, t_{0}\right],[D(A)]\right)$ is defined by $U(f)(\cdot)=j_{1} * C(\cdot) x+$ $D^{\alpha}(S * B f)(\cdot)$ on $\left[0, t_{0}\right]$ for all $f \in C\left(\left[0, t_{0}\right],[D(A)]\right)$, and

$$
M_{t_{0}}= \begin{cases}\sup _{0 \leq r \leq t_{0}}\|S(r)\|\left|A^{l} B\right|+\sum_{i=0}^{l-1} j_{2 i+1}\left(t_{0}\right) \| A^{i} B \mid & \text { if } 2 \leq \alpha \in \mathbb{N} \text { is even } \\ \sup _{0 \leq r \leq t_{0}}\|C(r)\|\|B\| & \text { if } \alpha=1 \\ \sup _{0 \leq r \leq t_{0}}\|C(r)\|\left|A^{l} B\right|+\sum_{i=0}^{l-1} j_{2 i+1}\left(t_{0}\right)\left|A^{i} B\right| & \text { if } 2 \leq \alpha \in \mathbb{N} \text { is odd } \\ \sup _{0 \leq r \leq t_{0}}\|\widetilde{C}(r)\||B| & \text { if } 0<\alpha<1 \\ \sup _{0 \leq r \leq t_{0}}\|\widetilde{C}(r)\|\left|A^{l} B\right|+\sum_{i=0}^{l-1} j_{2 i+1}\left(t_{0}\right)\left|A^{i} B\right| & \text { if }[\alpha]<\alpha<[\alpha]+1 \\ \sup _{0 \leq r \leq t_{0}}\|\widetilde{S}(r)\|\left|A^{l+1} B\right|+\sum_{i=0}^{l} j_{2 i+1}\left(t_{0}\right)\left|A^{i} B\right| & \text { if }[\alpha]<\alpha<[\alpha]+1 \\ & \text { and }[\alpha] \text { is odd. }\end{cases}
$$

Similarly, we can show that (3.5) also holds when $n \in \mathbb{N}$ is odd. Combining (3.2)(3.5), we can obtain that for each $x \in X$ there exists a unique function $w_{x}$ in $C\left(\left[0, T_{0}\right),[D(A)]\right)$ such that $w_{x}(\cdot)=j_{0} * S(\cdot) x+D^{\alpha} S * B w_{x}(\cdot)$ on $\left[0, T_{0}\right)$ as in the proof of Theorem 2.11, and then show that $u=j_{1} * w_{x}$ is the unique (strong) solution of $A C P\left(A, j_{\alpha+2}(\cdot) x+j_{1} * B w_{x}, 0,0\right)$ in $C^{2}\left(\left[0, T_{0}\right), X\right)$, so that $u=j_{1} * w_{x}$ is the unique (strong) solution of $A C P\left(A+B, j_{\alpha+2}(\cdot) x, 0,0\right)$ in $C^{2}\left(\left[0, T_{0}\right), X\right)$. Hence $A+B$ generates a nondegenerate local $(\alpha+2)$-times integrated cosine function $\widetilde{\widetilde{T}}(\cdot)$ on $X$ satisfying

$$
\widetilde{\widetilde{T}}(\cdot) x=j_{0} * S(\cdot) x+D^{\alpha} S * B \widetilde{\widetilde{T}}(\cdot) x \quad \text { on }\left[0, T_{0}\right)
$$

for all $x \in X$. We obtain from the assumption $B x \in D\left(A^{l+1}\right)$ for all $x \in D(A)$ and (2.8) that $\widetilde{\widetilde{T}}(\cdot) x$ is twice continuously differentiable on $\left[0, T_{0}\right)$ for all $x \in X$, and so $T(\cdot)$ defined by $T(t) x=\frac{d}{d t} \widetilde{T}(t) x$ for all $x \in X$ and $0 \leq t<T_{0}$, is a nondegenerate local $\alpha$-times integrated cosine function on $X$ with generator $A+B$ satisfying $T(\cdot) x=$ $C(\cdot) x+D^{\alpha+2} S * B \widetilde{\widetilde{T}}(\cdot) x$ on $\left[0, T_{0}\right)$ for all $x \in X$. Here $\widetilde{T}(t) x=\frac{d}{d t} \widetilde{\widetilde{T}}(t) x$ for all $x \in X$ and $0 \leq t<T_{0}$. Clearly, $S(\cdot)$ is exponentially Lipschitz continuous if $C(\cdot)$ is exponentially bounded. Applying Lemma 2.8, (2.10) and (3.6), we also get that $\widetilde{T}(\cdot)$ is exponentially Lipschitz continuous, if $C(\cdot)$ is exponentially bounded : it suffices to set $Y=[D(A)], V(\cdot)=S(\cdot), Z(\cdot)=\widetilde{\widetilde{T}}(\cdot)$ and 


$$
W(\cdot)= \begin{cases}C(\cdot) A^{l} B+\sum_{i=0}^{l-1} j_{2 i}(\cdot) A^{i} B & \text { if } \alpha \in \mathbb{N} \text { is even } \\ S(\cdot) A^{l+1} B+\sum_{i=0}^{l} j_{2 i}(\cdot) A^{i} B & \text { if } \alpha \in \mathbb{N} \text { is odd }\end{cases}
$$

in Lemma 2.8. This implies that $T(\cdot)$ is also exponentially bounded if $C(\cdot)$ is. Next if $C(\cdot)$ is norm continuous (resp., exponentially Lipschitz continuous). Applying Lemma 2.8 again, we get that $\underset{\widetilde{T}}{\widetilde{\widetilde{T}}}(\cdot)$ is also norm continuous (resp., exponentially Lipschitz continuous), and so $A^{i} B \widetilde{\widetilde{T}}(\cdot)$ for $0 \leq i \leq l+1$ are norm continuous (resp., exponentially Lipschitz continuous). Combining this with (2.10), we have that $D^{\alpha+2} S * B \widetilde{\widetilde{T}}(\cdot)$ is norm continuous (resp., exponentially Lipschitz continuous), which together with (3.1) implies that $T(\cdot)$ is also norm continuous (resp., exponentially Lipschitz continuous).

Theorem 3.2. Let $C(\cdot)$ be a locally Lipschitz continuous nondegenerate local $\alpha$ times integrated cosine function on $X$ with generator A for some $\alpha \geq 1$. Assume that $B$ is a bounded linear operator from $[D(A)]$ into $X$ such that $A+B$ is a closed linear operator from $D(A)$ into $X$. Then $A+B$ generates a locally Lipschitz continuous nondegenerate local $\alpha$-times integrated cosine function $T(\cdot)$ on $X$ satisfying (3.1) when

$$
\left\{\begin{array}{c}
R(B) \subset D\left(A^{l}\right) \text { if }[\alpha] \text { is even with } \alpha=[\alpha] \\
R(B) \subset D\left(A^{l+1}\right) \text { if }[\alpha] \text { is odd or }[\alpha] \text { is even with }[\alpha]<\alpha<[\alpha]+1 .
\end{array}\right.
$$

Proof. Just as in the proof of Theorem 3.1, we consider only the case $\alpha=$ $k-1 \in \mathbb{N}$, and so for each $0<t_{0}<T_{0}$, we deduce from Remark 2.12 and the fact $\left(C * A^{l} B f\right)^{\prime}(\cdot)=A\left(S * A^{l} B f\right)(\cdot)+j_{l-1} * A^{l} B f(\cdot)$ that $A+B$ generates a nondegenerate local $\alpha$-times integrated cosine function $T(\cdot)$ on $X$ satisfying (3.1). Clearly, $\widetilde{T}(\cdot)$ is locally Lipschitz continuous and $\widetilde{\widetilde{T}}(0)=0$ on $X$. It follows that $A^{i} B \widetilde{\widetilde{T}}(\cdot)$ is also locally Lipschitz continuous and $A^{i} B \widetilde{T}(0)=0$ on $X$ for all integers $0 \leq i \leq(l+1)$. Combining this with the local Lipschitz continuity of $C(\cdot)$, we conclude from Remark 2.12 that $\left(C * A^{l} B \widetilde{\widetilde{T}}\right)^{\prime}(\cdot)$ is locally Lipschitz continuous, which together with (2.10) that of $f$ is replaced by $\widetilde{\widetilde{T}}(\cdot)$, and (3.1) implies that $T(\cdot)$ is also locally Lipschitz continuous.

Corollary 3.3. Let $C(\cdot)$ be a nondegenerate local $\alpha$-times integrated cosine function on $X$ with generator $A$. Assume that $B$ is a bounded linear operator from $[D(A)]$ into $X$ such that $\rho(A+B)$ is nonempty. Then $A+B$ generates a nondegenerate local $\alpha$-times integrated cosine function $T(\cdot)$ on $X$ satisfying (3.1) for all $x \in X$ when

$$
\left\{\begin{array}{l}
R(B) \subset D\left(A^{l+1}\right) \text { if }[\alpha] \text { is even or }[\alpha] \text { is odd with } \alpha=[\alpha] \\
R(B) \subset D\left(A^{l+2}\right) \text { if }[\alpha] \text { is odd with }[\alpha]<\alpha<[\alpha]+1 .
\end{array}\right.
$$


Moreover, $T(\cdot)$ is also exponentially bounded (resp., norm continuous or exponentially Lipschitz continuous) if $C(\cdot)$ is.

Corollary 3.4. Let $C(\cdot)$ be a locally Lipschitz continuous nondegenerate local $\alpha$ times integrated cosine function on $X$ with generator $A$ for some $\alpha \geq 1$. Assume that $B$ is a bounded linear operator from $[D(A)]$ into $X$ such that $\rho(A+B)$ is nonempty. Then $A+B$ generates a locally Lipschitz continuous nondegenerate local $\alpha$-times integrated cosine function $T(\cdot)$ on $X$ satisfying (3.1) when

$$
\left\{\begin{array}{c}
R(B) \subset D\left(A^{l}\right) \text { if }[\alpha] \text { is even with } \alpha=[\alpha] \\
R(B) \subset D\left(A^{l+1}\right) \text { if }[\alpha] \text { is odd or }[\alpha] \text { is even with }[\alpha]<\alpha<[\alpha]+1 .
\end{array}\right.
$$

The conclusion of Theorem 3.1 is still true when the assumption that $A+B$ is a closed linear operator from $D(A)$ into $X$ is replaced by assuming that $A B=B A$ on $D\left(A^{2}\right)$.

Corollary 3.5. Let $C(\cdot)$ be a nondegenerate local $\alpha$-times integrated cosine function on $X$ with generator $A$. Assume that $B$ is a bounded linear operator from $[D(A)]$ into $X$ such that $A B=B A$ on $D\left(A^{2}\right)$. Then $A+B$ generates a nondegenerate $\alpha$-times integrated cosine function $T(\cdot)$ on $X$ satisfying

$$
T(\cdot) x=C(\cdot) x+D^{\alpha} S *(\lambda-A) B(\lambda-A)^{-1} T(\cdot) x \quad \text { on }\left[0, T_{0}\right)
$$

for all $x \in X$ when

$$
\left\{\begin{array}{l}
R(B) \subset D\left(A^{l+1}\right) \text { if }[\alpha] \text { is even or }[\alpha] \text { is odd with } \alpha=[\alpha] \\
R(B) \subset D\left(A^{l+2}\right) \text { if }[\alpha] \text { is odd with }[\alpha]<\alpha<[\alpha]+1 .
\end{array}\right.
$$

Here $\lambda \in \rho(A)$ is fixed. Moreover, $T(\cdot)$ is also exponentially bounded (resp., norm continuous or exponentially Lipschitz continuous) if $C(\cdot)$ is.

It is easy to see from the assumption $A B=B A$ on $D\left(A^{2}\right)$ that $A+B$ is closed (see [18]). Just as in the proof of Theorem 2.11, we also have that for each $x \in X$ there exists a unique function $w_{x}$ in $C\left(\left[0, T_{0}\right), X\right)$ such that $w_{x}=C(\cdot) x+D^{\alpha} S *(\lambda-$ A) $B(\lambda-A)^{-1} w_{x}$, and so $j_{1} * w_{x}$ is the unique solution of

$$
\begin{aligned}
& A C P\left(A, j_{\alpha} x+j_{1} *(\lambda-A) B(\lambda-A)^{-1} w_{x}, 0,0\right) \\
= & A C P\left(A, j_{\alpha} x+(\lambda-A) B(\lambda-A)^{-1} j_{1} * w_{x}, 0,0\right) \\
= & A C P\left(A, j_{\alpha} x+(\lambda-A) B(\lambda-A)^{-1} j_{1} * w_{x}, 0,0\right) \\
= & A C P\left(A, j_{\alpha} x+B(\lambda-A)(\lambda-A)^{-1} j_{1} * w_{x}, 0,0\right) \\
= & A C P\left(A, j_{\alpha} x+B j_{1} * w_{x}, 0,0\right) \\
= & A C P\left(A, j_{\alpha} x+B j_{1} * w_{x}, 0,0\right)
\end{aligned}
$$

in $C^{2}\left(\left[0, T_{0}\right), X\right)$. Hence $u=j_{1} * w_{x}$ is the unique function in $C^{2}\left(\left[0, T_{0}\right), X\right)$ such that $u^{\prime \prime}=A u+j_{\alpha} x+B u=(A+B) u+j_{\alpha} x$ on $\left[0, T_{0}\right)$ and $u(0)=0=u^{\prime}(0)$. Applying 
Theorem 2.6 again, we get that $A+B$ generates a nondegenerate local $\alpha$-times integrated cosine function on $X$ satisfying (3.7) which is defined by $T(\cdot) x=w_{x}(\cdot)$ for all $x \in X$. Moreover, $T(\cdot)$ is also exponentially bounded (resp., norm continuous or exponentially Lipschitz continuous) if $S(\cdot)$ is.

By slightly modifying the proof of Theorem 2.13, the next unbounded perturbation result concerning locally Lipschitz continuous local $\alpha$-times integrated cosine functions on $X$ is also attained.

Corollary 3.6. Let $C(\cdot)$ be a nondegenerate locally Lipschitz continuous local $\alpha$-times integrated cosine function on $X$ with generator $A$ for some $\alpha \geq 1$. Assume that $B$ is a bounded linear operator from $[D(A)]$ into $X$ such that $A B=B A$ on $D\left(A^{2}\right)$. Then $A+B$ generates a nondegenerate locally Lipschitz continuous local $\alpha$-times integrated cosine function $T(\cdot)$ on $X$ satisfying (3.7) when

$$
\left\{\begin{array}{c}
R(B) \subset D\left(A^{l}\right) \text { if }[\alpha] \text { is even with } \alpha=[\alpha] \\
R(B) \subset D\left(A^{l+1}\right) \text { if }[\alpha] \text { is odd or }[\alpha] \text { is even with }[\alpha]<\alpha<[\alpha]+1 .
\end{array}\right.
$$

We end this paper with a simple illustrative example. Let $X=L^{p}\left(\mathbb{R}^{n}\right)$ for some $1 \leq p<\infty$ and $n \in \mathbb{N}$, and $\Delta$ denote the Laplacian operator with maximal distribution domain. It is known that $\Delta$ generates an exponentially bounded nondegenerate $\alpha$-times integrated cosine function on $X$ for all $\alpha>(n-1)\left|\frac{1}{p}-\frac{1}{2}\right|$ with $\overline{D(\Delta)}=\overline{W^{p, 2}\left(\mathbb{R}^{n}\right)}=X$ (see [5]), and so $\Delta$ generates an exponentially Lipschitz continuous $(\alpha+1)$-times integrated cosine function $C(\cdot)$ on $L^{\infty}\left(\mathbb{R}^{n}\right)$ and $\overline{D(\Delta)}=C_{0}\left(\mathbb{R}^{n}\right)$. Applying Theorem 2.13 , we get that $\Delta+B$ generates a nondegenerate locally Lipschitz continuous local $\beta$-times integrated cosine function $T(\cdot)$ on $L^{\infty}\left(\mathbb{R}^{n}\right)$ satisfying (2.8) when $\frac{n+1}{2}<\beta \leq 2$ and $B: C_{0}\left(\mathbb{R}^{n}\right) \rightarrow L^{\infty}\left(\mathbb{R}^{n}\right)$ is a bounbed linear operator from $C_{0}\left(\mathbb{R}^{n}\right)$ into $L^{\infty}\left(\mathbb{R}^{n}\right)$ defined by $B(f)(x)=\int_{\mathbb{R}^{n}} f(x-y) d \mu(y)$ for all $f \in C_{0}\left(\mathbb{R}^{n}\right)$ and $x \in \mathbb{R}^{n}$. Here $\mu$ is a fixed finite regular Borel measure on $\mathbb{R}^{n}$.

\section{REFERENCES}

1. W. Arendt, C. J. K. Batty, H. Hieber and F. Neubrander, Vector-Valued Laplace Transforms and Cauchy Problems, Vol. 96, Birkhauser Verlag, Basel-Boston-Berlin, 2001.

2. W. Arendt and H. Kellermann, Integrated Solutions of Volterra Integrodifferential Equations and Applications, Pitman Res. Notes Math., Longman, Harlow, 190 (1989), 21-51.

3. H. O. Fattorini, Second Order Linear Differential Equations in Banach Spaces, in: NorthHolland Math. Stud., 108, North-Holland, Amsterdam, 1985.

4. J. A. Goldstein, Semigroups of Linear Operators and Applications, Oxford, 1985.

5. M. Hieber, Integrated Semigroups and Differential Operators on $L^{p}, \mathrm{PhD}$. Dissertation, Tubingen, 1989.

6. M. Kostic, Distribution Cosine Functions, Taiwanese J. Math., 10 (2006), 739-775. 
7. C.-C. Kuo and S.-Y. Shaw, C-Cosine Functions and the Abstract Cauchy Problem I, II, J. Math. Anal. Appl., 210 (1997), 632-646, 647-666.

8. C.-C. Kuo, On $\alpha$-Times Integrated C-Cosine Functions and Abstract Cauchy Problem I, J. Math. Anal. Appl., 313 (2006), 142-162.

9. C.-C. Kuo, On Exponentially Bounded $\alpha$-Times Integrated C-Cosine Functions, Yokohama Math. J., 52 (2005), 59-72.

10. C.-C. Kuo, On Existence and Approximation of Solutions of Second Order Abstract Cauchy Problem, Taiwanese J. Math., 14 (2010), 1093-1110.

11. C.-C. Kuo, Perturbation Theorems for Local Integrated Semigroups, Studia Math., 197 (2010), 13-26.

12. C.-C. Kuo, On Local Integrated C-Cosine Function and Weak Solution of Second Order Abstract Cauchy Problem, Taiwanese J. Math., 14 (2010), 2027-2042.

13. S.-Y. Shaw and Y.-C. Li, On Generators of Integrated C-Semigroups and C-Cosine Functions, Semigroup Forum, 47 (1993), 29-35.

14. J. E. Shi and W. X. Zheng, On Local Integrated Cosine Functions, Northeast Math. J., 17 (2001), 21-26.

15. T. Takenaka and S. Piskarev, Local C-Cosine Families and N-Times Integrated Local Cosine Families, Taiwanese J. Math., 8 (2004), 515-546.

16. C. C. Travis and G. F. Webb, Perturbation of Strongly Continuous Cosine Family Generators, Colleq. Math., 45 (1981), 277-285.

17. S. W. Wang and Z. Huang, Strongly Continuous Integrated C-Cosine Operator Functions, Studia Math., 126 (1997), 273-289.

18. S. W. Wang, M. Y. Wang and Y. Shen, Perturbation Theorems for Local Integrated Semigroups and Their Applications, Studia Math., 170 (2005), 121-146.

19. T. J. Xiao and J. Liang, The Cauchy Problem for Higher-Order Abstract Differential Equations, Lectures Notes in Math., 1701, Springer, 1998.

20. Q. Zheng, Integrated Cosine Functions, Internat. J. Math. and Math. Sci., 19 (1996), $575-580$.

21. J. Zheng and Q. Zheng, On $\alpha$-Times Integrated Cosine Functions, Mathematical Japonica, 50 (1999), 401-408.

22. Q. Zheng, Coercive Differential Operators and Fractionally Integral Cosine Functions, Taiwanese J. Math., 6 (2002), 59-65.

Chung-Cheng Kuo

Department of Mathematics

Fu Jen University

New Taipei City 24205, Taiwan

E-mail: cckuo@math.fju.edu.tw 